\title{
OSCILLATION REGION OF A PIECEWISE-SMOOTH MODEL OF THE VOCAL FOLDS*
}

\author{
JORGE C. LUCERO ${ }^{\dagger}$ AND CRISTIANE A. GAJO $\ddagger$
}

\begin{abstract}
The two-mass model of the vocal folds is a popular representation of their dynamical structure used in phonation studies. This paper presents an analysis of a recent piecewise-smooth version of the model. This version has two equilibrium positions, and in one of them (the initial prephonatory position) the system is nondifferentiable. Standard methods of stability analysis do not apply for that position, because they require smoothness of the system. A geometrical approach is applied instead, which is an extension of a method previously developed for planar systems. The analysis shows the existence of a transcritical bifurcation between the equilibrium positions, and a Hopf bifurcation related to each of them. The oscillation region of the model is next determined as the area delimited by the Hopf bifurcations. The results are illustrated by a bifurcation diagram and trajectory plots.
\end{abstract}

Key words. Oscillation, two-mass model, vocal folds, bifurcation, piecewise smooth

AMS subject classifications. 34C15, 34C23, 34C25, 34C60, 37G10, 70K42, 92C10

\section{Introduction}

The two-mass model of the human vocal folds [8] has been widely used to simulate their oscillatory motion at phonation. Besides its capability to produce realistic synthesis of voice [11] and patterns of glottal aerodynamics [22], its simplicity allows for analytical treatments of the vocal folds' biodynamics. For example, it has been applied to studies of oscillation regions and phonation threshold conditions [3, 14, 19, 25], irregular and pathological vibrations $[7,9,10,18,25]$, voice registers $[1,15]$, hysteresis patterns of phonation onset-offsets [16], and prosthesis design [13]. It has been even extended to other oscillatory biomechanical systems, such as the vocal membranes of primates and bats [17], and the lips when playing a brass musical instrument [31].

In voice and speech synthesis applications, the model is used as a glottal sound source coupled to some vocal tract representation. The vocal tract may be represented as a straight tube of varying cross-sectional area, which is approximated by a finite number of concatenated cylindrical sections [5, 27]. The acoustical properties of the set of sections are next modelled by using a transmission line analogy terminated in a radiation load for the lips (e.g. $[8,11,16]$ ), or a wave propagation approach (e.g. $[13,24,26])$. The first approach is the preferred technique in articulatory speech synthesis [5].

Since the original version of the two-mass model presented by Ishizaka and Flanagan more than three decades ago [8], several modifications and updates of its equations have been proposed. Some of the new versions have incorporated better descriptions of the tissue biomechanical properties [11, 13, 16, 26] and the glottal aerodynamics $[3,13,22,25]$, with various degrees of complexity. Particularly, Steinecke and Herzel's version [25] has proven useful. Their model is derived from a large simplification of the original equations, in order to reduce it to its basic oscillatory principle. Thus,

\footnotetext{
*Received: January 13, 2006; accepted (in revised version): April 18, 2006. Communicated by Jack Xin.

This work was supported by CT-Info/MCT/CNPq and Pronex/FAPDF (Brazil).

${ }^{\dagger}$ Department of Mathematics, University of Brasilia, Brasilia DF 70910-900, Brazil (lucero@ mat.unb.br).

${ }^{\ddagger}$ Faculty of Mathematics, Rio Verde University, Rio Verde GO 75900-000, Brazil (cristianegajo@ yahoo.com.br).
} 
while the original version included more than ten coupled second order differential equations, Steinecke and Herzel's version consists of only two, with far fewer parameters. For that reason, it has been preferred in studies on phonation dynamics $[6,7,9,10,18,30,32]$, where the focus is on characterizing the main oscillatory mechanism under various normal and pathological configurations, rather than performing detailed simulations of the vocal fold motion or realistic speech synthesis. This paper will explore the dynamics of that version by analyzing its equilibrium positions, bifurcations, and generation of periodic motions. The main purpose is to determine the oscillation region of the model. Precise knowledge of that region is relevant for vocalization and speech production studies, because it characterizes laryngeal conditions required for phonation.

Let us note here that the model is a piecewise-smooth dynamical system. In such systems, the phase space may be divided into a number of regions, in which the system's vector field is smooth [4]. At the boundary between smooth regions, the vector field is continuous but nondifferentiable. Such systems arise in a variety of applications, and their analysis constitutes an exciting field of current research, e.g. $[12,21,33]$. In fact, nonsmoothness may cause a whole new rich family of bifurcations not existing in smooth systems, collectively denoted as $C$-bifurcations [4]. Due to nondifferentiability, the analysis of such bifurcations prevents the use of standard local methods (which require smoothness of the system, e.g., [23]), and thus demands the development of new techniques. As it will be shown later, the vocal fold model studied here has an equilibrium position at the boundary between two smooth regions, and in that boundary the vector field is nondifferentiable. In Steinecke and Herzel's work [25], stability and bifurcations related to that position were analyzed by the standard technique of computing the eigenvalues of the system's Jacobian, which actually would not apply. Here, we will use a geometrical approach recently used to detect Hopf bifurcations in piecewise-smooth planar systems [12, 21, 33].

The present work follows a previous analysis of the original version of the two-mass model [14]. That analysis showed regions of existence of three equilibrium positions, a stable limit cycle, and several bifurcation phenomena: saddle-node, Hopf, and transcritical. A secondary purpose of this work is to update those results with a more recent version of the model.

\section{The two-mass model}

2.1. Main equations. Fig. 2.1 shows a sketch of the model. Each vocal fold is represented by two mass-damper-spring systems $\left(m_{1}-r_{1}-k_{1}\right.$ and $\left.m_{2}-r_{2}-k_{2}\right)$, coupled through a spring $\left(k_{c}\right)$. The two folds are assumed identical, and they move symmetrically with respect to the glottal midline, in the horizontal direction. The opposite vocal folds may collide during their motion, closing the glottis and consequently interrupting the airflow.

For clarity in the present analysis, the derivation of the model's equations are briefly reviewed.

When the glottis is open, the equations of motion may be written as

$$
\left\{\begin{array}{l}
m_{1} \ddot{x_{1}}+r_{1} \dot{x_{1}}+k_{1} x_{1}+k_{c}\left(x_{1}-x_{2}\right)=f_{1}\left(x_{1}, x_{2}\right), \\
m_{2} \ddot{x_{2}}+r_{2} \dot{x_{2}}+k_{2} x_{2}+k_{c}\left(x_{2}-x_{1}\right)=f_{2}\left(x_{1}, x_{2}\right),
\end{array}\right.
$$

where $x_{i}, i=1,2$, is the horizontal displacement of mass $m_{i}, r_{i}$ is the damping coefficient, $k_{i}$ and $k_{c}$ are the stiffness coefficients, and $f_{i}\left(x_{1}, x_{2}\right)$ is the force produced by action of the airflow. When the masses are at their initial prephonatory position 


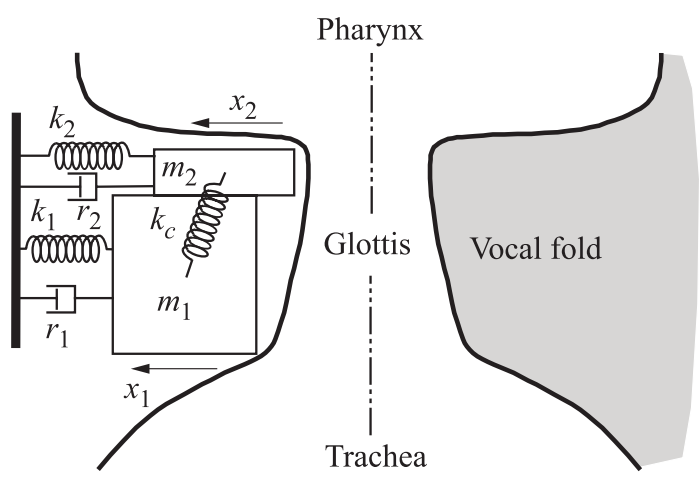

FIG. 2.1. The two-mass model [8].

$\left(x_{i}=0\right)$, they are at a distance $x_{0}$ from the glottal midline. Then, each mass $i$ collides with its opposite counterpart at a displacement $x_{i}=-x_{0}$. Collision forces are modeled by increasing the related stiffness coefficient by an amount $c_{i}$.

The glottal aerodynamics is modeled by neglecting all effects of the vocal tract, and assuming that the subglottal pressure (at the entry of the larynx) is constant and equal to the lung pressure $P_{L}$, and the supraglottal pressure (at the exit of the larynx) is the atmospheric pressure. These are standard assumptions to investigate the main mechanism of the vocal fold oscillation, isolated from vocal tract and subglottal influences, and approximately correspond to laboratory conditions of an excised larynx $[2]$.

Bernoulli flow is assumed until the narrowest region of the glottis. Downstream this region, and because of the abrupt area expansion, the flow detaches from the glottal wall and forms a jet stream where all energy is lost due to turbulence [22]. Let $P_{i}(i=1,2)$ be the glottal pressure at the position of mass $m_{i}$, and $a_{i}=2 l_{g}\left(x_{i}+x_{0}\right)$ be the cross-sectional glottal area at that position, where $l_{g}$ is the vocal fold length (perpendicular to the airflow direction).

When the glottal channel is rectangular or convergent, i.e., $a_{1} \geq a_{2}$, the airflow is assumed to detach from the glottal wall at the upper border of mass $m_{2}$. The application of Bernoulli's equation yields

$$
P_{L}=P_{1}+\frac{\rho u_{g}^{2}}{2 a_{1}^{2}}=P_{2}+\frac{\rho u_{g}^{2}}{2 a_{2}^{2}}
$$

where $\rho$ is the air density, and $u_{g}$ the volume velocity of glottal airflow. Since all the airflow energy is lost at the glottal exit, $P_{2}=0$, and substituting in the above equations we obtain

$$
P_{1}=P_{L}\left(1-\frac{a_{2}^{2}}{a_{1}^{2}}\right)
$$

When the glottal channel is divergent, i.e. $a_{1}<a_{2}$, the flow detaches from the glottal wall at the boundary between both masses. In this case, the glottal pressure vanishes and $P_{1}=P_{2}=0$.

The aerodynamic forces acting on the masses are finally computed as $f_{i}=d_{i} l_{g} P_{i}$ $(i=1,2)$, where $d_{i}$ is the height (in the direction of the airflow) of each mass $m_{i}$. 
When the opposite masses collide, the glottis closes and interrupts the airflow. In this case, the forces on the masses are assumed to be

$$
\left\{\begin{array}{cl}
f_{1}=d_{1} l_{g} P_{L}, & \text { if } a_{1}>0, a_{2} \leq 0 \\
=0 & \text { if } a_{1} \leq 0 .
\end{array}\right.
$$

and $f_{2}=0$ in all cases $\left(a_{1} \leq 0\right.$ or $\left.a_{2} \leq 0\right)$.

The above equations may be conveniently written by defining a "switching" function of the glottal cross-sectional area $a$

$$
\Theta(a)= \begin{cases}\tanh \left[50 a / a_{0}\right], & a>0 \\ 0, & a \leq 0\end{cases}
$$

where $a_{0}=2 l_{g} x_{0}$ is the area at the prephonatory position of the vocal folds (i.e., when $\left.x_{1}=x_{2}=0\right)$.

The complete equations of motion are then

$$
\left\{\begin{array}{l}
m_{1} \ddot{x_{1}}+r_{1} \dot{x_{1}}+k_{1} x_{1}+\Theta\left(-a_{1}\right) c_{1}\left(x_{1}+x_{0}\right)+k_{c}\left(x_{1}-x_{2}\right)=d_{1} l_{g} P_{1}, \\
m_{2} \ddot{x_{2}}+r_{2} \dot{x_{2}}+k_{2} x_{2}+\Theta\left(-a_{2}\right) c_{2}\left(x_{2}+x_{0}\right)+k_{c}\left(x_{2}-x_{1}\right)=0,
\end{array}\right.
$$

and

$$
P_{1}=P_{L}\left[1-\Theta\left(a_{\min }\right)\left(\frac{a_{\min }}{a_{1}}\right)^{2}\right] \Theta\left(a_{1}\right)
$$

where $a_{\min }=\min \left(a_{1}, a_{2}\right)$.

All parameters are non-negative, and the following values represent the standard configuration of the model for normal voicing of a male adult: $m_{1}=0.125 \mathrm{~g}, m_{2}=0.025$ $\mathrm{g}, k_{c}=25 \mathrm{~N} / \mathrm{m}, k_{1}=80 \mathrm{~N} / \mathrm{m}, k_{2}=8 \mathrm{~N} / \mathrm{m}, c_{1}=3 k_{1}, c_{2}=3 k_{2}, r_{1}=r_{2}=0.02 \mathrm{~N} \mathrm{~s} / \mathrm{m}$, $l_{g}=1.4 \mathrm{~cm}, d_{1}=0.25 \mathrm{~cm}, P_{L}=800 \mathrm{~Pa}[8]$.

Details on the derivation of the above equations may be easily found in Steinecke and Herzel's paper [25], and other references cited above.

2.2. Normalization. The equations of motion are next normalized, to reduce the number of parameters. The above parameter values show that the lower system (see Fig. 2.1) is thicker and more massive than the upper one. This system may be considered as a representation of the main muscular body of the vocal folds [15]. In such a case, the upper system $m_{2}-r_{2}-k_{2}$ would represent the mucosa and other superficial tissues of the vocal folds. We select the lower system as a reference for the normalization, and express all parameters relative to it, as follows: First, an adimensional measure of time $\tau=\left(\sqrt{k_{1} / m_{1}}\right) t$ is adopted, which expresses time relative to the natural period of the lower system. Replacing the temporal derivatives in (2.6) and simplifying factors results in

$$
\left\{\begin{array}{l}
x_{1}^{\prime \prime}+\frac{r_{1}}{\sqrt{m_{1} k_{1}}} x_{1}^{\prime}+x_{1}+\frac{c_{1}}{k_{1}} \Theta\left(-a_{1}\right)\left(x_{1}+x_{0}\right)+\frac{k_{c}}{k_{1}}\left(x_{1}-x_{2}\right)=\frac{d_{1} l_{g} P_{1}}{k_{1}}, \\
\frac{m_{2}}{m_{1}} x_{2}^{\prime \prime}+\frac{r_{2}}{\sqrt{m_{1} k_{1}}} x_{2}^{\prime}+\frac{k_{2}}{k_{1}} x_{2}+\frac{c_{2}}{k 1} \Theta\left(-a_{2}\right)\left(x_{2}+x_{0}\right)+\frac{k_{c}}{k_{1}}\left(x_{2}-x_{1}\right)=0
\end{array}\right.
$$

where the derivative is relative to $\tau$. A natural choice for normalized parameters seems to be: $\alpha_{2}=m_{2} / m_{1}, \beta_{1}=r_{1} / \sqrt{m_{1} k_{1}}, \beta_{2}=r_{2} / \sqrt{m_{1} k_{1}}, \gamma_{2}=k_{2} / k_{1}, \delta=k_{c} / k_{1}, \eta_{1}=$ $c_{1} / k_{1}, \eta_{2}=c_{2} / k_{1}$. Displacements are normalized relative to the prephonatory position, 
by introducing the new variables $u_{1}=x_{1} / x_{0}, u_{2}=x_{2} / x_{0}$. Finally, the normalized subglottal pressure is defined as $p=d_{1} l_{g} P_{l} /\left(k_{1} x_{0}\right)$.

The normalized equations of motion become

$$
\left\{\begin{aligned}
u_{1}^{\prime \prime}+\beta_{1} u_{1}^{\prime}+u_{1}+\theta\left(-u_{1}-1\right) \eta_{1}\left(u_{1}+1\right)+\delta\left(u_{1}-u_{2}\right) & =p f_{p}\left(u_{1}, u_{2}\right), \\
\alpha_{2} u_{2}^{\prime \prime}+\beta_{2} u_{2}^{\prime}+\gamma_{2} u_{2}+\theta\left(-u_{2}-1\right) \eta_{2}\left(u_{2}+1\right)+\delta\left(u_{2}-u_{1}\right) & =0,
\end{aligned}\right.
$$

with

$$
\begin{gathered}
f_{p}\left(u_{1}, u_{2}\right)=\left[1-\theta\left(u_{\min }+1\right)\left(\frac{u_{\min }+1}{u_{1}+1}\right)^{2}\right] \theta\left(u_{1}+1\right), \\
\theta(u)= \begin{cases}\tanh (50 u) & , u>0 \\
0, & u \leq 0\end{cases}
\end{gathered}
$$

and $u_{\min }=\min \left(u_{1}, u_{2}\right)$.

The above equation (2.9) shows that the upper and lower systems are coupled through both mechanical and aerodynamical factors, which are controlled by parameters $\delta$ and $p$, respectively. Parameter $\delta$ is related to the tissue stiffness coefficients, and so depends on the muscular settings of the larynx [29]. Parameter $p$ is related to the lung pressure $P_{L}$, which is the main control for voicing onset and intensity. In the next sections, we will explore the behavior of the system when varying those coupling parameters.

Let us note also that the above system is piecewise-smooth. The plane $u_{1}-u_{2}$ may be divided into the five regions shown in Fig. 2.2, which will be denoted as regions I $\left(u_{1} \geq u_{2}>-1\right)$, II $\left(u_{2} \geq u_{1}>-1\right)$, III $\left(u_{1} \leq-1\right.$ and $\left.u_{2}>-1\right)$, IV $\left(u_{1} \leq-1\right.$ and $\left.u_{2} \leq-1\right)$, and $\mathrm{V}\left(u_{1}>-1\right.$ and $\left.u_{2} \leq-1\right)$. Within each region the system is smooth, and at the border lines it is continuous but non-differentiable.

The standard configuration of the normalized parameters is: $\alpha_{2}=0.2, \beta_{1}=\beta_{2}=$ $0.2, \gamma=0.1, \delta=0.3125, \eta_{1}=3, \eta_{2}=0.3, p=1.75$. Figures 2.3 and 2.4 show the output of the model for those parameters.

\section{Equilibrium positions}

In regions I and II (open glottis), $u_{1}>-1, u_{2}>-1$. In this case, $\theta\left(-u_{1}-1\right)=$ $\theta\left(-u_{2}-1\right)=0$. It may be safely assumed that $\theta\left(u_{1}+1\right) \approx 1$, and $\theta\left(u_{\min }+1\right) \approx 1$. The approximations hold over almost all the oscillatory cycle except during the brief intervals when the vocal folds are almost in contact each other (e.g., accepting a $1 \%$ error, the approximations will be valid for $\left.u_{1}, u_{\min }>-0.9998\right)$.

In region I $\left(u_{2} \leq u_{1}\right)$, the equations of motion are

$$
\left\{\begin{aligned}
\left.u_{1}^{\prime \prime}+\beta_{1} u_{1}^{\prime}+u_{1}+\delta\left(u_{1}-u_{2}\right)\right) & =p\left[1-\left(\frac{u_{2}+1}{u_{1}+1}\right)^{2}\right] \\
\alpha_{2} u_{2}^{\prime \prime}+\beta_{2} u_{2}^{\prime}+\gamma_{2} u_{2}+\delta\left(u_{2}-u_{1}\right) & =0 .
\end{aligned}\right.
$$

The equilibrium positions $\left(u_{1}^{*}, u_{2}^{*}\right)$ are obtained by setting all derivates to zero in the above equations, which produces

$$
\begin{aligned}
u_{1}^{*}+\delta\left(u_{1}^{*}-u_{2}^{*}\right) & =p\left[1-\left(\frac{u_{2}^{*}+1}{u_{1}^{*}+1}\right)^{2}\right], \\
\gamma_{2} u_{2}^{*}+\delta\left(u_{2}^{*}-u_{1}^{*}\right) & =0 .
\end{aligned}
$$




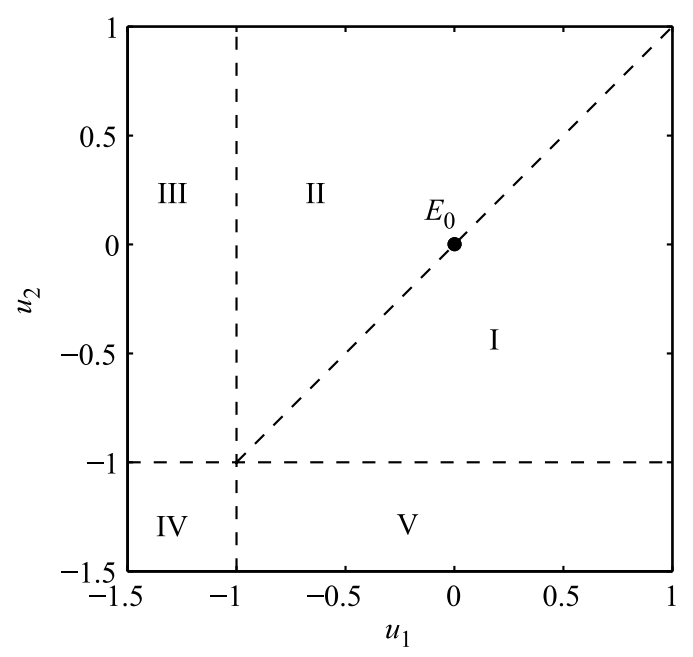

FIG. 2.2. Regions of smoothness of the two-mass model. In regions I and II, the glottis is open, and the glottal channel has convergent and divergent shapes, respectively. In regions III, $I V$, and $V$, the glottis is closed. At the border lines between regions, the system is continuous but non-differentiable. $E_{0}=(0,0)$ is the prephonatory position of the vocal folds.

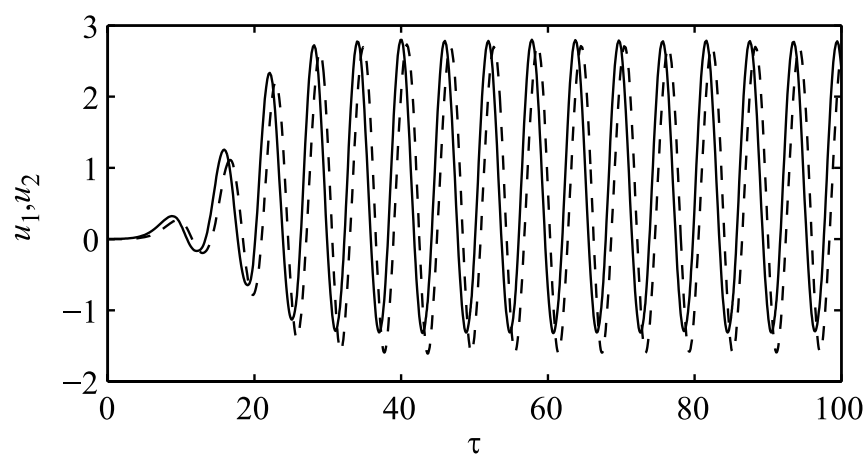

FIG. 2.3. Solution curves for the standard configuration. Full line: $u_{1}(\tau)$, dashed line: $u_{2}(\tau)$.

From (3.3) we obtain the relation

$$
u_{2}^{*}=\frac{\delta}{\gamma_{2}+\delta} u_{1}^{*}
$$

This relation may be used to eliminate $u_{2}^{*}$ from $(3.2)$, which yields

$$
\left(1+\frac{\gamma_{2} \delta}{\gamma_{2}+\delta}\right) u_{1}^{*}=p \frac{\gamma_{2}}{\gamma_{2}+\delta} \frac{\left[2+u_{1}^{*}+\delta u_{1}^{*} /\left(\gamma_{2}+\delta\right)\right]}{\left(u_{1}^{*}+1\right)^{2}} u_{1}^{*}
$$

Clearly, one solution is $u_{1}^{*}=u_{2}^{*}=0$, which is the prephonatory position of the vocal folds. This position will be denoted as $E_{0}=(0,0)$. The other solutions are given by 


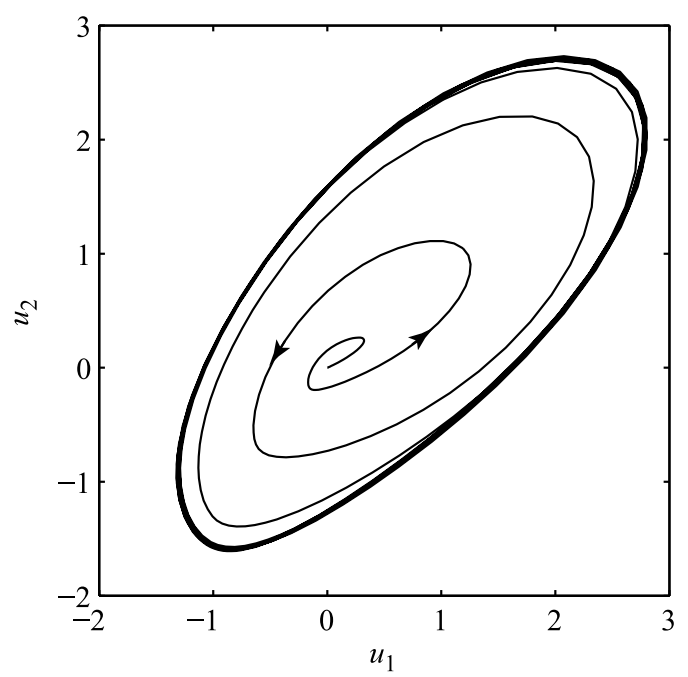

FIG. 2.4. Trajectory for the standard configuration.

the following quadratic equation in $\left(u_{1}^{*}+1\right)$

$$
\left(1+\frac{\gamma_{2} \delta}{\gamma_{2}+\delta}\right)\left(u_{1}^{*}+1\right)^{2}-p \frac{\gamma_{2}}{\gamma_{2}+\delta}\left(1+\frac{\delta}{\gamma_{2}+\delta}\right)\left(u_{1}^{*}+1\right)-p\left(\frac{\gamma_{2}}{\gamma_{2}+\delta}\right)^{2}=0
$$

In this equation, the coefficients of the second and third terms are negative, which indicates the existence of two real roots of opposite sign. The negative solution is not valid, since it implies $u_{1}^{*}<-1$ and hence does not belong to the open glottis condition. The second solution is $u_{1}^{*}>-1$ and hence $u_{2}^{*}>-1$. Note that (3.4) implies that $\left|u_{2}^{*}\right| \leq\left|u_{1}^{*}\right|$. Hence, in order to have the initial assumption of $u_{2} \leq u_{1}$, the equilibrium position must satisfy $u_{1}^{*} \geq 0$ (and so $u_{1}^{*} \geq u_{2}^{*} \geq 0$ ). This second position will be denoted by $E_{1}=\left(u_{1}^{*}, u_{2}^{*}\right)$.

For some combination of parameter values, $E_{1}$ may become coincident with $E_{0}$. Letting $u_{1}^{*}=u_{2}^{*}=0$ in (3.6), we obtain

$$
1+\frac{\gamma_{2} \delta}{\gamma_{2}+\delta}=2 p \frac{\gamma_{2}}{\gamma_{2}+\delta}
$$

In region II $\left(u_{2}>u_{1}\right), u_{\min }=u_{1}$, which produces $f_{p}\left(u_{1}, u_{2}\right) \equiv 0$. The equations of motion become the linear system

$$
\left\{\begin{aligned}
u_{1}^{\prime \prime}+\beta_{1} u_{1}^{\prime}+u_{1}+\eta_{1}\left(u_{1}+1\right)+\delta\left(u_{1}-u_{2}\right) & =0 \\
\alpha_{2} u_{2}^{\prime \prime}+\beta_{2} u_{2}^{\prime}+\gamma_{2} u_{2}+\delta\left(u_{2}-u_{1}\right) & =0
\end{aligned}\right.
$$

with a unique equilibrium position at $E_{0}=(0,0)$.

In regions III, IV, and V (closed glottis), $u_{1} \leq-1$ or $u_{2} \leq-1$. Let us consider region III $\left(u_{1} \leq-1\right.$ and $\left.u_{2}>-1\right)$. In this case, $\theta\left(-u_{1}-1\right) \approx 1, \theta\left(-u_{2}-1\right)=0, \theta\left(u_{1}+\right.$ $1)=0$, and $f_{p}\left(u_{1}, u_{2}\right)=0$. The equations of motion become a linear system similar to (3.8), and has a unique equilibrium position at $E_{0}=(0,0)$. However, this position is outside region III and therefore not valid. Similar results are obtained for the other two regions IV and $\mathrm{V}$. 


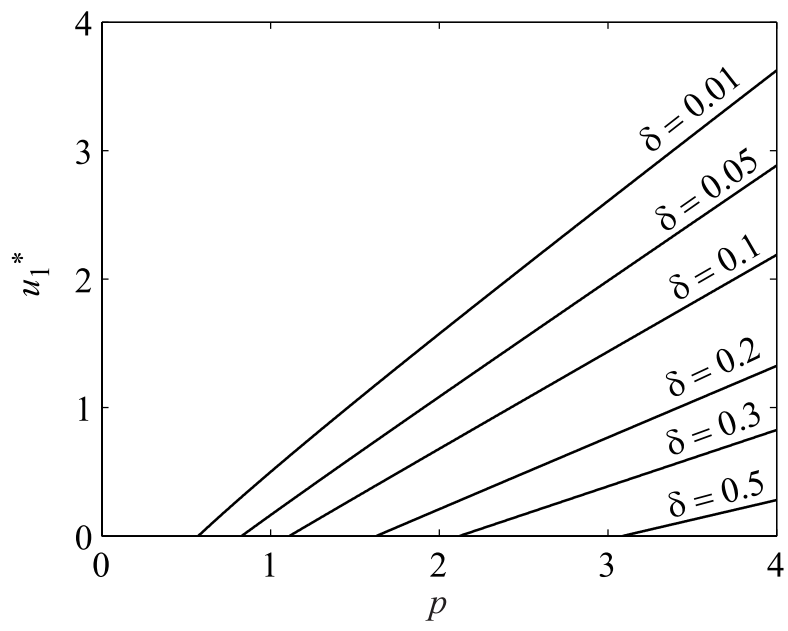

FIG. 3.1. $u_{1}^{*}$ for the second equilibrium position $E_{1}$ vs. $p$, and various values of $\delta$. Other parameters are fixed at their standard values.

In summary, the system has two equilibrium positions: the prephonatory position $E_{0}=(0,0)$, and a second equilibrium position $E_{1}=\left(u_{1}^{*}, u_{2}^{*}\right)$ given by $(3.4)$ and $(3.6)$, and under the condition $u_{1}^{*} \geq u_{2}^{*} \geq 0$.

Figure 3.1 shows plots of $u_{1}^{*}$ for $E_{1}$. As shown by the plots, the second position exists for $p$ larger than a critical value, at which $u_{1}^{*}=0$.

Some differences may already be noted between this model and the original version of the two-mass model, which is smooth over the whole open glottis regions (regions I and II). In that version, a third equilibrium position besides $E_{0}$ and $E_{1}$ exists, for some configuration of parameter values [14]. Further, position $E_{1}$ also exists in region II.

\section{Stability analysis}

4.1. Equilibrium position $E_{1}=\left(u_{1}^{*}, u_{2}^{*}\right)$. Let us consider first equilibrium position $E_{1}=\left(u_{1}^{*}, u_{2}^{*}\right)$. This position is located in region $\mathrm{I}$, in which the equations of motion are given by (3.1). Letting $v_{1}=u_{1}^{\prime}, v_{2}=u_{2}^{\prime}$, the equations may be written as

$$
\left\{\begin{aligned}
u_{1}^{\prime} & =v_{1} \\
v_{1}^{\prime} & \left.=-\beta_{1} v_{1}-u_{1}-\delta\left(u_{1}-u_{2}\right)\right)+p\left[1-\left(\frac{u_{2}+1}{u_{1}+1}\right)^{2}\right] \\
u_{2}^{\prime} & =v_{2} \\
v_{2}^{\prime} & =\frac{1}{\alpha_{2}}\left[-\beta_{2} v_{2}-\gamma_{2} u_{2}-\delta\left(u_{2}-u_{1}\right)\right]
\end{aligned}\right.
$$

The Jacobian at the equilibrium positions is

$$
J\left(u_{1}^{*}, 0, u_{2}^{*}, 0\right)=\left[\begin{array}{cccc}
0 & 1 & 0 & 0 \\
p D_{1}-1-\delta & -\beta_{1} & p D_{2}+\delta & 0 \\
0 & 0 & 0 & 1 \\
\delta / \alpha_{2} & 0 & -\left(\gamma_{2}+\delta\right) / \alpha_{2} & -\beta_{2} / \alpha_{2}
\end{array}\right]
$$


where

$$
\begin{aligned}
& D_{1}=2\left(u_{2}^{*}+1\right)^{2} /\left(u_{1}^{*}+1\right)^{3} \\
& D_{2}=-2\left(u_{2}^{*}+1\right) /\left(u_{1}^{*}+1\right)^{2} .
\end{aligned}
$$

The eigenvalues $\lambda$ are the roots of the characteristic equation

$$
\begin{aligned}
& \lambda^{4}+\left(\beta_{1}+\beta_{2}\right) \lambda^{3}+\left(1+\delta-p D_{1}+\frac{\gamma_{2}+\delta}{\alpha_{2}}+\frac{\beta_{1} \beta_{2}}{\alpha_{2}}\right) \lambda^{2}+ \\
& \frac{1}{\alpha_{2}}\left[\beta_{1}\left(\gamma_{2}+\delta\right)+\beta_{2}\left(1+\delta-p D_{1}\right)\right] \lambda+ \\
& \frac{1}{\alpha_{2}}\left[\left(1+\delta-p D_{1}\right)\left(\gamma_{2}+\delta\right)-\delta\left(\delta+p D_{2}\right)\right]=0
\end{aligned}
$$

which has the general form $\lambda^{4}+b_{1} \lambda^{3}+b_{2} \lambda^{2}+b_{3} \lambda+b_{4}=0$.

A change of stability occurs when one or more roots cross the imaginary axes, and two cases are of interest here: a real root crossing the imaginary axes at $b_{4}=0$, and a pair of complex roots crossing the imaginary axes at $b_{1} b_{2} b_{3}-b_{3}^{2}-b_{1}^{2} b_{4}=0[20]$.

Let us assume that $p$ has a large value so that the equilibrium position $E_{1}$ exists (see Fig. 3.1). If $p$ decreases, equilibrium position $E_{1}$ tends to the prephonatory position $E_{0}$. As seen in the previous section, both positions coalesce when $p$ takes the value given by (3.7). Substituting $u_{1}^{*}=u_{2}^{*}=0$ in (4.3) and (4.4) produces $D_{1}=-D_{2}=$ 2. Further, from (3.7), we obtain $2 p=1+\delta+\delta / \gamma_{2}$. Replacing those values into

$$
b_{4}=\left(1+\delta-p D_{1}\right)\left(\gamma_{2}+\delta\right)-\delta\left(\delta+p D_{2}\right)
$$

results in $b_{4}=0$. This result means that, as $E_{1}$ approaches and finally coalesces with the prephonatory position $E_{0}$, a real eigenvalue becomes zero. This fact signals the occurrence of a transcritical bifurcation, at which two equilibrium positions become coincident and increase or decrease the dimensions of their respective stable and unstable manifolds, in opposite directions [23]. That bifurcation was also found in the original version of the two-mass model [14]. In the present case, the bifurcation occurs exactly at the border line between regions I and II, and position $E_{1}$ does not exist in one side of the bifurcation (region II).

The case of a pair of complex roots crossing the imaginary axes indicates the occurrence of a Hopf bifurcation, at which the equilibrium position changes stability and a limit cycle is generated. Both the Hopf and the transcritical bifurcation will be further explored later in $\S 5$, with numerical examples.

4.2. Equilibrium position $E_{0}=(0,0)$. We consider next the stability of the prephonatory position $E_{0}=(0,0)$. Particularly, we are interested in the generation of a sustained oscillation from this position. Note that $E_{0}$ is located on the border line $u_{1}=u_{2}$ between regions I and II, at which the system is nondifferentiable. Classical analytical methods for detecting Hopf bifurcations do not apply here, since smoothness is a requisite for them. We follow instead a piecewise linear approach, e.g., [12, 21, 33]. In that approach, the equations of motion are replaced by a piecewise linear approximation for the vicinity of $E_{0}$.

In region I, the equations of motion are given by (3.1). The right side of the first equation is approximated by the linear terms of its Taylor expansion around $E_{0}=(0,0)$, given by $2 p\left(u_{1}-u_{2}\right)$. In region II, the equations of motion are given by 
the linear system (3.8). Thus, we consider the piecewise linear approximation

$$
\left\{\begin{aligned}
\left.u_{1}^{\prime \prime}+\beta_{1} u_{1}^{\prime}+u_{1}+\eta_{1}\left(u_{1}+1\right)+\delta\left(u_{1}-u_{2}\right)\right) & =p \widetilde{f}_{p}\left(u_{1}, u_{2}\right), \\
\alpha_{2} u_{2}^{\prime \prime}+\beta_{2} u_{2}^{\prime}+\gamma_{2} u_{2}+\delta\left(u_{2}-u_{1}\right) & =0
\end{aligned}\right.
$$

where

$$
\tilde{f}_{p}\left(u_{1}, u_{2}\right) \approx\left\{\begin{array}{lll}
2\left(u_{1}-u_{2}\right) & \text { for } u_{1} \geq u_{2} & (\text { region I) } \\
0 & \text { for } u_{1} \leq u_{2} & (\text { region II }) .
\end{array}\right.
$$

The characteristic equation for region $\mathrm{I}$ is

$$
\begin{aligned}
& \lambda^{4}+\left(\beta_{1}+\beta_{2}\right) \lambda^{3}+\left(1+\delta-2 p+\frac{\gamma_{2}+\delta}{\alpha_{2}}+\frac{\beta_{1} \beta_{2}}{\alpha_{2}}\right) \lambda^{2}+ \\
& \frac{1}{\alpha_{2}}\left[\beta_{1}\left(\gamma_{2}+\delta\right)+\beta_{2}(1+\delta-2 p)\right] \lambda+ \\
& \frac{1}{\alpha_{2}}\left[(1+\delta-2 p)\left(\gamma_{2}+\delta\right)-\delta(\delta-2 p)\right]=0
\end{aligned}
$$

and for region II is

$$
\begin{aligned}
& \lambda^{4}+\left(\beta_{1}+\beta_{2}\right) \lambda^{3}+\left(1+\delta+\frac{\gamma_{2}+\delta}{\alpha_{2}}+\frac{\beta_{1} \beta_{2}}{\alpha_{2}}\right) \lambda^{2}+ \\
& \frac{1}{\alpha_{2}}\left[\beta_{1}\left(\gamma_{2}+\delta\right)+\beta_{2}(1+\delta)\right] \lambda+ \\
& \frac{1}{\alpha_{2}}\left[(1+\delta)\left(\gamma_{2}+\delta\right)-\delta^{2}\right]=0
\end{aligned}
$$

Note that the characteristic equation (4.9) may be obtained from (4.8) just by letting $p=0$.

We want to determine the conditions for the existence of a periodic solution around $E_{0}$. Note first that in region II the system behaves as a pair of coupled damped oscillators (all the roots of 4.9 have negative real parts).

Fig. 4.1 shows numerical examples of the roots of the characteristic equation (4.8), when varying $p$ from 0 to 2 , and $\delta$ from 0.1 to 1 . When $p=0$, the four roots are complex and have negative real parts. As $p$ increases, the pair of complex roots closest to the imaginary axes $\left(\lambda_{1}\right.$ and $\left.\lambda_{2}\right)$ moves toward it and eventually crosses it taking positive real parts. The other pair of roots $\left(\lambda_{3}\right.$ and $\left.\lambda_{4}\right)$, on the other hand, moves away from the imaginary axes to the left, increasing the magnitude of their (negative) real parts.

When the system is in region II, its behavior is determined by the eigenvalues at the position corresponding to $p=0$. Let us denote them as $\lambda_{i}^{\mathrm{II}}$. Now assume, as an approximation, that any trajectory in region II is determined by the eigenvalues closest to the imaginary axes $\lambda_{1,2}^{\mathrm{II}}=\zeta_{a}^{\mathrm{II}} \pm i \omega_{a}^{\mathrm{II}}$ (with $\zeta_{a}^{\mathrm{II}}<0$ ), and hence it may expressed by

$$
\begin{aligned}
& u_{1}(\tau) \approx A_{1} e^{\zeta_{a}^{\mathrm{II}} \tau} \cos \left(\omega_{a}^{\mathrm{II}} \tau-\theta_{1}\right) \\
& u_{2}(\tau) \approx A_{2} e^{\zeta_{a}^{\mathrm{II}} \tau} \cos \left(\omega_{a}^{\mathrm{II}} \tau-\theta_{2}\right) .
\end{aligned}
$$

The other eigenvalues, $\lambda_{3,4}^{\mathrm{II}}=\zeta_{b}^{\mathrm{II}} \pm i \omega_{b}^{\mathrm{II}}$, would produce similar trajectory components as above. However, since $\zeta_{b}^{\mathrm{II}}<\zeta_{a}^{\mathrm{II}}<0$, those components would decrease in magnitude 


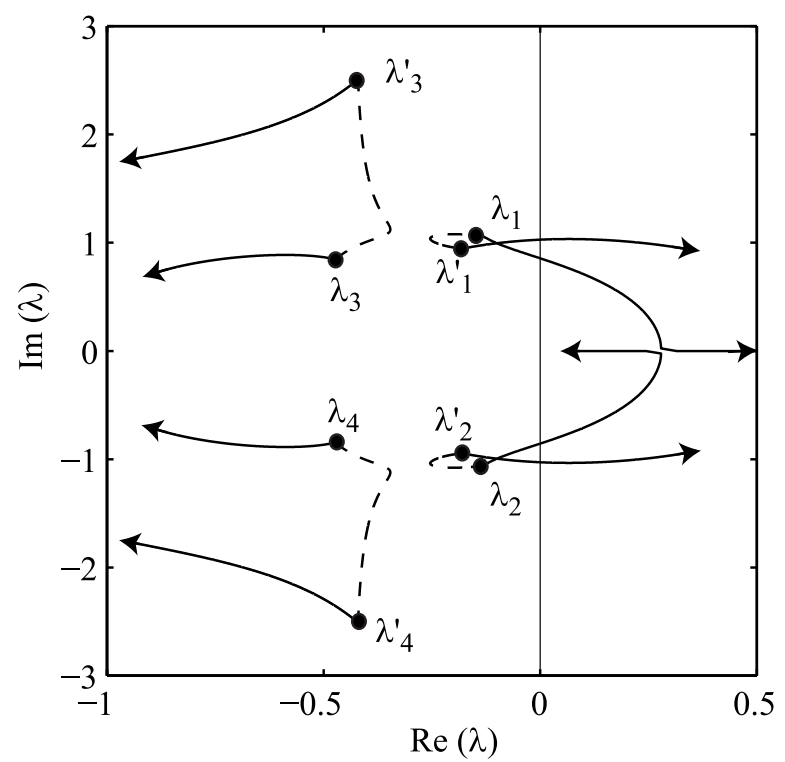

FIG. 4.1. Roots of the characteristic equation (4.8) for region I, when varying $p$ from 0 (black circles) to 2 (arrowheads), and $\delta$ from $0.1\left(\lambda_{i}\right)$ to $1\left(\lambda_{i}^{\prime}\right)$. The black circles, for $p=0$, also represent the location of the roots for region II.

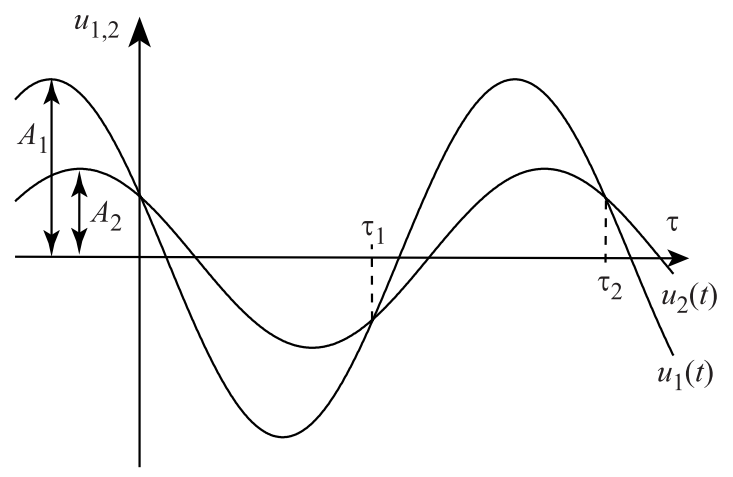

FIG. 4.2. Assumed solutions for $u_{1}(\tau)$ and $u_{2}(\tau)$.

at a faster rate than the components related to $\lambda_{1,2}^{\mathrm{II}}$ and rapidly become negligible. Then, we assume that $\left|\zeta_{b}^{\mathrm{II}}\right|$ is large enough so that their influence on the trajectories may be neglected. In case of the example in Fig. 4.1, the ratio $\left|\zeta_{b}^{\mathrm{II}} / \zeta_{a}^{\mathrm{II}}\right|$ of the real part of the eigenvalues is in the range 1.4 to 3.7 , so the above assumption might seem somewhat crude. However, it is adopted just as a first approximation, which will be verified later through numerical resolution of the differential equations.

Let us also state the initial conditions to be such that $u_{1}(0)=u_{2}(0)=u_{0}, \theta_{2}>$ $\theta_{1}>0$, and $\tau_{1}=\pi / \omega_{a}$ the next value of $\tau$ at which $u_{1}\left(\tau_{1}\right)=u_{2}\left(\tau_{1}\right)$ (see Fig. 4.2). Then, both $u_{1}(\tau)$ and $u_{2}(\tau)$ are within region II for $\tau \in\left[0, \tau_{1}\right]$. At $\tau=\tau_{1}$ we have $u_{1}\left(\tau_{1}\right)=u_{2}\left(\tau_{1}\right)=-u_{0} e^{2 \pi \zeta_{a}^{\mathrm{II}} / \omega_{a}^{\mathrm{II}}}$. 


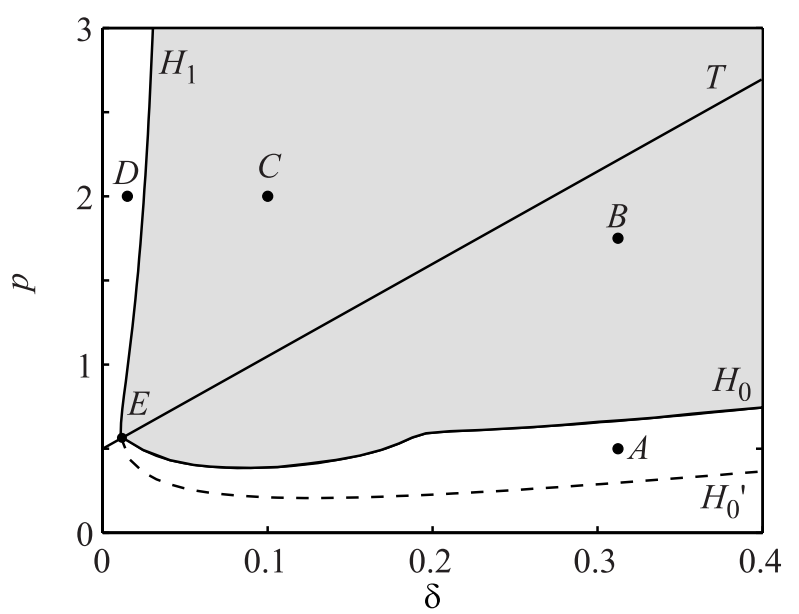

FIG. 5.1. Bifurcation diagram. $T$ is a transcritical bifurcation, $H_{0}$ and $H_{1}$ are Hopf bifurcations for equilibrium positions $E_{0}$ and $E_{1}$, respectively, and $H_{0}^{\prime}$ is a Hopf bifurcation assuming smoothness of the equations at $E_{0}$ (see explanation in text). The gray area is the oscillation region of the model.

From $\tau=\tau_{1}$ until $\tau=\tau_{2}$, which is the next point at which $u_{1}\left(\tau_{2}\right)=u_{2}\left(\tau_{2}\right)$, the system is within region $\mathrm{I}$. We assume that $p$ has a positive value such that the corresponding eigenvalues $\lambda_{1}^{\mathrm{I}}$ and $\lambda_{2}^{\mathrm{I}}$ have positive real parts, with $\lambda_{1,2}^{\mathrm{I}}=\zeta_{a}^{\mathrm{I}} \pm i \omega_{a}^{\mathrm{I}}$, and $\zeta_{a}^{\mathrm{I}}>0$. Again, we assume that the trajectories in this region are determined by $\lambda_{1,2}^{\mathrm{I}}$, and repeating the same previous reasoning, we find that $u_{1}\left(\tau_{2}\right)=u_{2}\left(\tau_{2}\right)=$ $u_{0} e^{2 \pi\left(\zeta_{a}^{\mathrm{I}} / \omega_{a}^{\mathrm{I}}+\zeta_{a}^{\mathrm{II}} / \omega_{a}^{\mathrm{II}}\right)}$. The condition for a periodic solution is then $u_{1}(0)=u_{1}\left(\tau_{2}\right)$ (and similarly for $u_{2}$ ), which yields

$$
\frac{\zeta_{a}^{\mathrm{I}}}{\omega_{a}^{\mathrm{I}}}+\frac{\zeta_{a}^{\mathrm{II}}}{\omega_{a}^{\mathrm{II}}}=0
$$

The above condition matches results of a more formal analysis on a planar system [33]. That analysis considered a piecewise-smooth planar system with a fixed point at a corner of $n$ smooth subsystems. Assuming that the Jacobian of each subsystem has complex eigenvalues, a bifurcation function of a control parameter $p$ is defined as $B(p)=\sum_{i=1}^{n} \psi^{i} \zeta^{i}(p) / \omega^{i}(p)$, were $\psi^{i}$ is the corner's angle for subsystem $i$, and $\zeta^{i}(p)$ and $\omega^{i}(p)$ are the real and imaginary parts of the corresponding eigenvalues. A generalized Hopf bifurcation occurs when $B(p)=0$, and $B^{\prime}(p) \neq 0$, at which the fixed point changes stability and a periodic orbit is produced. The case of $n=1$ reduces to the standard Hopf bifurcation in smooth systems, and the case of $n=2$ with $\psi^{1}=\psi^{2}=\pi$ (i.e., when the boundary between the smooth subregions is a line), is similar to (4.11). We assume that this condition also holds for the present system, under the simplifying assumptions noted above.

\section{Bifurcation diagram}

Using the results of the previous section, the bifurcation diagram shown in Fig. 5.1 is obtained. The diagram was constructed with $\delta$ and $p$ as control parameters, and keeping all other parameters fixed at their standard values.

The straight line $T$ is the location of the transcritical bifurcation, given by (3.7). At this bifurcation, both equilibrium positions $E_{0}$ and $E_{1}$ are coincident, as explained 
in $\S 4.1$. Below this line, equilibrium position $E_{1}$ does not exist.

Curve $H_{1}$ is a Hopf bifurcation for equilibrium position $E_{1}$. This position is stable at the left side of the curve, and unstable at the right side. A limit cycle is generated at this bifurcation, when crossing the curve from left to right.

Curve $H_{0}$ is a Hopf bifurcation for equilibrium position $E_{0}$. The curve was computed as explained in $\S 4.2$, using the piecewise linear approximation. Position $E_{0}$ is stable below the curve and unstable above it, and a limit cycle is generated when crossing the curve in the upward direction.

Point $E$ is a codimension-2 bifurcation, and corresponds to two roots of the characteristic equation (4.9) equal to zero.

The straight line $T$ and curves $H_{0}$ and $H_{1}$ divide the diagram into 4 regions. Below $H_{0}$, and below $T$ at the left of point $E$, there is a unique equilibrium position, $E_{0}$, and it is stable. Fig. 5.2 shows a trajectory in the $u_{1}-u_{2}$ plane for parameter values corresponding to point $A$. The trajectory is a spiral of decreasing amplitude, indicating a stable position at the origin $(0,0)$.

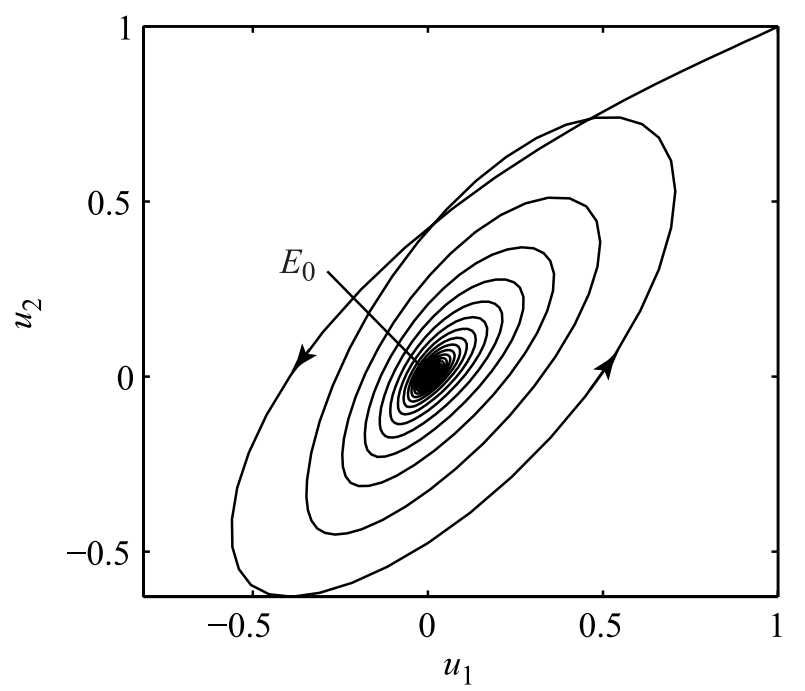

FIG. 5.2. Trajectory in the $u_{1}-u_{2}$ plane, for $p=0.5, \delta=0.3125$ (point $A$ in Fig. 5.1)

Between $H_{0}$ and $T, E_{0}$ is also the only equilibrium position, and it is unstable. The plots already shown in Fig. 2.3 and 2.4, simulating a normal adult male phonation, correspond to parameter values at point $B$.

Between $H_{1}$ and $T$, there are two equilibrium positions, $E_{0}$ and $E_{1}$, both unstable. Fig. 5.3 shows a trajectory for parameter values corresponding to point $C$.

Finally, to the left of $H_{1}$ and above $T$, at the left of the bifurcation diagram, both $E_{0}$ and $E_{1}$ exists, and they are unstable and stable, respectively. Fig. 5.4 shows a trajectory for parameters values corresponding to point $D$, starting in the vicinity of $E_{0}$. Note that the system moves away from $E_{0}$, and spirals toward position $E_{1}$.

The oscillation region of the model is then the region limited by the Hopf bifurcations, above $H_{0}$ and to the right of $H_{1}$ (gray area in Fig. 5.1). Both limits indicate that a certain level of coupling, both mechanical (represented by parameter $\delta$ ) and aerodynamical (represented by parameter $p$ ), is required to produce an oscillation. If 


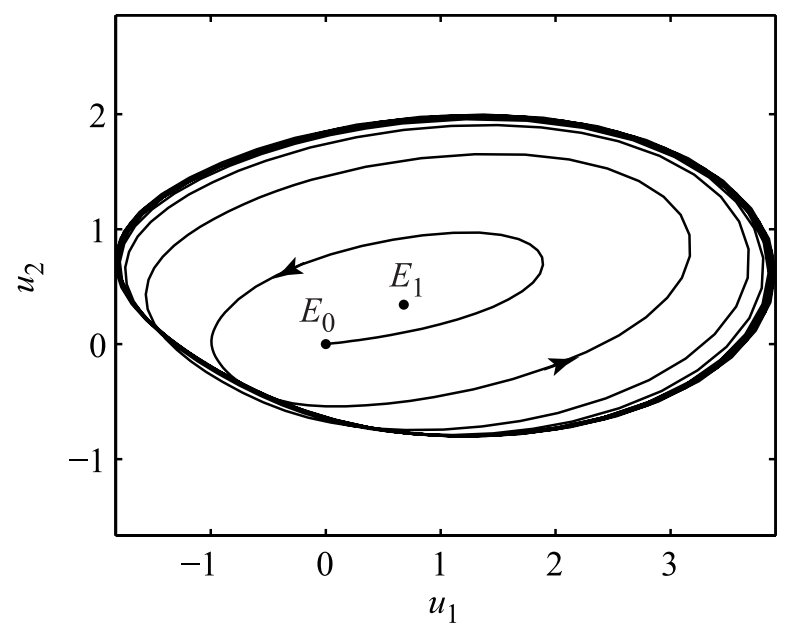

FIG. 5.3. Trajectory in the $u_{1}-u_{2}$ plane, for $p=2, \delta=0.1$ (point $C$ in Fig. 5.1)

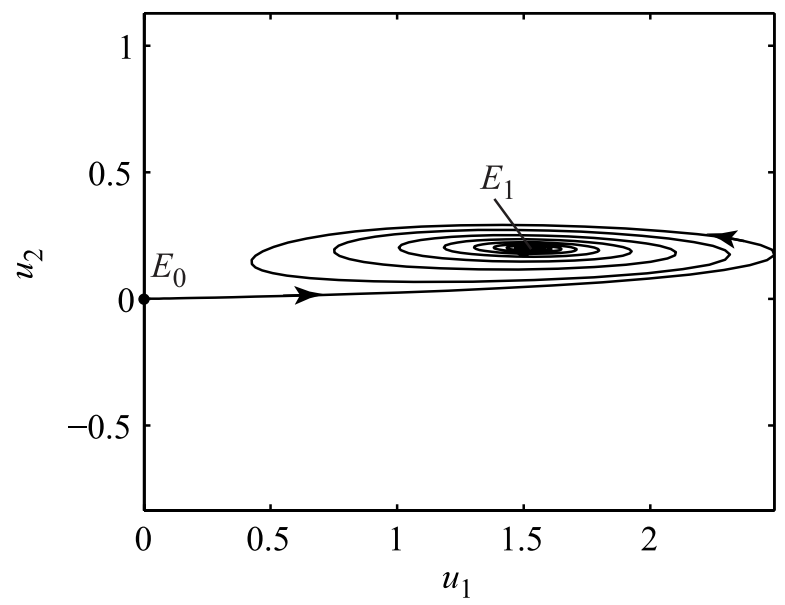

FIG. 5.4. Trajectory in the $u_{1}-u_{2}$ plane, for $p=2, \delta=0.015$ (point D in Fig. 5.1)

the mechanical coupling $\delta$ is low enough, then the upper mass-damper-spring system tends to behave as a simple damped oscillator, which inhibits the production of a sustained oscillation. The lower aerodynamical limit, given by $H_{0}$, is well known in phonation studies, and is called phonation threshold pressure [28]. At this threshold, the energy transferred from the airflow to the vocal folds overcomes the energy lost in the tissues by dissipation, and consequently a self-excited oscillation may start.

In curve $H_{0}$, there is a region around $\delta=0.2$ where $p$ has a steeper increment. This region corresponds precisely to the range of $\delta$ values at which the eigenvalues $\lambda_{1,2}$ become closer to $\lambda_{3,4}$ (see Fig. 4.1). Recall that the Hopf bifurcation for $H_{0}$ was computed under the assumption that the real parts of $\lambda_{3,4}$ were negative and large enough in magnitude so that their effect could be neglected. In this region, the 
assumption is not well satisfied, which implies a larger error when computing the Hopf bifurcation points.

Next, let us assume that the model equations for region I given by (3.1), also hold for region II. In that case, the system is smooth in the vicinity of equilibrium position $E_{0}=(0,0)$. The Hopf bifurcation for $E_{0}$ may be then determined by the standard method of detecting a pair of complex roots of the characteristic equation (4.8) crossing the imaginary axis. In this case, the bifurcation would occur at curve $H_{0}^{\prime}$ in Fig. 5.1. This is in fact the method used in previous works [25], which results in bifurcation values about half the correct values. The error is clearly evident by the trajectory plot in Fig. 5.2 (point $A$ in Fig. 5.1): although the system is above curve $H_{0}^{\prime}, E_{0}$ is a stable position and no limit cycle is produced.

The difference between thresholds given by $H_{0}^{\prime}$ and $H_{0}$ may be understood in physical terms. Consider an oscillation around $E_{0}$. In the first case, the aerodynamical force (left side of 3.1), acts over the whole oscillatory cycle. It is this force which fuels the oscillation, by transferring energy from the airflow to the tissues. In the second case, the aerodynamic force acts only during half of the cycle, when the glottal channel is convergent (region I). When it is divergent (region II), the aerodynamic force is zero (see equations 3.8), and there is no transfer of energy from the airflow. The net energy transferred over a whole cycle, therefore, is about half the amount that would correspond to the first case. To compensate, the threshold value of pressure $p$ has to approximately double.

\section{Conclusion}

This article has explored the dynamical structure of a non-smooth version of the two-mass model of the vocal folds. It has shown the existence of two equilibrium positions, both of which may undergo Hopf bifurcations and produce a limit cycle. There is also a transcritical bifurcation at which both positions coalesce, and one of them disappears from the system. The oscillation region of the model was determined as the area limited by the Hopf bifurcations.

Those results are, in general, similar to those obtained from the original version of the model [14]. Main differences appear in the number of equilibrium positions (three in the original model), and in the location of the Hopf bifurcation for the prephonatory equilibrium position, caused by the non-smooth nature of the current version.

The present analysis is also a correction to a previous work [25], in which the non-smoothness of the model was disregarded when computing the oscillation conditions. The equations of the current model are nondifferentiable precisely at the equilibrium position which represents the initial prephonatory condition of the vocal folds. Stability of this position was determined here by using a piecewise linear technique developed for planar systems. The application of this technique has been done under simplifying assumptions, which produce only approximate results. However, it has shown that the value of the air pressure required to produce the oscillation is higher (about double) than the value previously computed.

As noted by a reviewer of this paper, nondifferentiability at $u_{1}=-1$, and $u_{2}=-1$ (i.e., at the collision of the opposite vocal folds) may be easily eliminated by adopting a switching function $\theta(u)$ differentiable at $u=0$. However, such a change would have no effect in the results of the present analysis, since the equilibrium positions are located in regions I and II (in the open glottis region). Further, the line of nondifferentiability at $u_{1}=u_{2}$, which separates both regions and contains equilibrium position $E_{0}$, would still persist.

This article also had the intention of contributing to the subject of analysis of 
$C$-bifurcations in nonsmooth dynamical systems. Further research efforts should be dedicated to the development of a computational method for bifurcation analysis in such systems. Particularly, extensions of the piecewise linear technique applied here to higher dimensional systems would be useful.

\section{REFERENCES}

[1] D. Berry, Mechanisms of modal and nonmodal phonation, J. Phonetics, 29, 431-450, 2001.

[2] D. A. Berry, H. Herzel, I. R. Titze and B. H. Story, Bifurcations in excised larynx experiments, J. Voice, 10, 129-138, 1996.

[3] M. P. de Vries, H. K. Schutte, A. E. P. Veldman and G. J. Verkerke, Glottal flow through a two-mass model: Comparison of Navier-Stokes solutions with simplified models, J. Acoust. Soc. Am., 111, 1847-1853, 2002.

[4] M. di Bernardo, M. I. Feigin, S. J. Hogan and M. E. Homer, Local analysis of C-bifurcations in $n$-dimensional piecewise-smooth dynamical system, Chaos, Solitons, and Fractals, 10, 1881-1908, 1999.

[5] D. G. Childers, Speech Processing and Synthesis Toolboxes, John Wiley Sons, New York, 2000.

[6] M. Döllinger, U. Hoppe, F. Hettlich, J. Lohscheller, S. Schuberth and U. Eysholdt, Vibration parameter extraction from endoscopic image series of the vocal folds, IEEE Trans. Biomed. Eng., 49, 773-781, 2002.

[7] H. Herzel and C. Knudsen, Bifurcations in a vocal fold model, Nonlinear Dynamics, 7, 53-64, 1995.

[8] K. Ishizaka and J. L. Flanagan, Synthesis of voiced sounds from a two-mass model of the vocal folds, Bell Systems Technical Journal, 51, 1233-1268, 1972.

[9] J. J. Jiang and Y. Zhang, Chaotic vibration induced by turbulent noise in a two-mass model of the vocal folds, J. Acoust. Soc. Am., 112, 2127-2133, 2002.

[10] J. J. Jiang, Y. Zhang and J. Stern, Modeling of chaotic vibrations in symmetric vocal folds, J. Acoust. Soc. Am., 110, 2120-2128, 2001.

[11] T. Koizumi, S. Taniguchi, and S. Hiromitsu, Two-mass models of the vocal cords for natural sounding voice synthesis, J. Acoust. Soc. Am., 82, 1179-1192, 1987.

[12] T. Küpper and S. Moritz, Generalized Hopf bifurcation for non-smooth planar systems, Philos. Trans. R. Soc. Lond. Ser. A, 359, 2483-2496, 2001.

[13] N. J. C. Lous, G. C. J. Hofmans, R. N. J. Veldhuis and A. Hirschberg, A symmetrical two-mass vocal-fold model coupled to vocal tract and trachea, with application to prosthesis design, Acta Acustica, 84, 1135-1150, 1998.

[14] J. C. Lucero, Dynamics of the two-mass model of the vocal folds: Equilibria, bifurcations, and oscillation region, J. Acoust. Soc. Am., 94, 3104-3111, 1993.

[15] J. C. Lucero Chest- and falsetto-like oscillations in a two-mass model of the vocal folds, J. Acoust. Soc. Am., 100, 3355-3359, 1996.

[16] J. C. Lucero and L. L. Koenig, Simulations of temporal patterns of oral airflow in men and women using a two-mass model of the vocal folds under dynamic control, J. Acoust. Soc. Am., 117, 1362-1372, 2005.

[17] P. Mergell, W. T. Fitch and H. Herzel, Modeling the role of nonhuman vocal membranes in phonation, J. Acoust. Soc. Am., 105, 2020-2028, 1999.

[18] P. Mergell, H. Herzel and I. R. Titze, Irregular vocal fold vibration - High-speed observation and modeling, J. Acoust. Soc. Am., 108, 2996-3002, 2000.

[19] P. Mergell, H. Herzel, T. Wittenberg, M. Tigges and U. Eysholdt, Phonation onset: Vocal fold modeling and high-speed glottography, J. Acoust. Soc. Am., 104, 464-470, 1998.

[20] K. Ogata, Modern Control Engineering, Prentice-Hall, Englewood Cliffs, 1970.

[21] G. Olivar, F. Angulo and M. di Bernardo, Hopf-like transitions in nonsmooth dynamical systems, in Proc. 2004 International Symposium on Circuits and Systems, 4, 693-696, 2004.

[22] X. Pelorson, A. Hirschberg, R. R. van Hassel, A. P. J. Wijnands and Y. Auregan, Theoretical and experimental study of quasisteady-flow separation within the glottis during phonation. Application to a modified two-mass model, J. Acoust. Soc. Am., 96, 3416-3431, 1994.

[23] L. Perko, Differential Equations and Dynamical Systems, Springer-Verlag, New York, 1991.

[24] M. M. Sondhi and J. Schroeter, A hybrid time-frequency domain articulatory speech synthesizer, IEEE Trans. Acoust., Speech, and Signal Processing, ASSP-35, 955-967, 1987.

[25] I. Steinecke and H. Herzel, Bifurcations in an asymmetric vocal fold model, J. Acoust. Soc. Am., 97, 1878-1884, 1995.

[26] B. H. Story and I. R. Titze, Voice simulation with a body-cover model of the vocal folds, J. 
Acoust. Soc. Am., 97, 1249-1260, 1995.

[27] I. R. Titze, Principles of Voice Production, Prentice-Hall, Englewood Cliffs, 1994.

[28] I. R. Titze, The physics of small-amplitude oscillation of the vocal folds, J. Acoust. Soc. Am., 83, 1988.

[29] I. R. Titze and B. H. Story, Rules for controlling low-dimensional vocal fold models with muscle activation, J. Acoust. Soc. Am., 112, 1064-1076, 2002.

[30] I. Tokuda and H. Herzel, Detecting synchronizations in an asymmetric vocal fold model from time series data, Chaos, 15, 013702, 2005.

[31] C. E. Vilain, X. Pelorson, A. Hirschberg, L. Le Marrec, W. O. Root and J. Willems, Contribution to the physical modeling of the lips. Influence of the mechanical boundary conditions, Acta Acustica united with Acustica, 89, 882-887, 2003.

[32] Y. Zhang, J. Jiang and D. A. Rahn, Studying vocal fold vibrations in Parkinson's disease with a nonlinear model, Chaos, 15, 033903, 2005.

[33] Y. Zou and T. Kupper, Generalized Hopf bifurcation emanated from a corner for piecewise smooth planar system, Nonlinear Analysis, 62, 1-17, 2005. 